\title{
OPTIMAL POINT TO POINT PATH PLANNING OF FLEXIBLE MANIPULATOR UNDER LARGE DEFORMATION BY USING HARMONY SEARCH METHOD
}

\author{
HABIB ESFANDIAR \\ Department of Mechanical and Aerospace Engineering, Science and Research Branch, Islamic Azad University, Tehran, \\ Iran; e-mail: habibesfandiar2014@gmail.com

\section{Moharam Habibnejad Korayem} \\ Department of Mechanical and Aerospace Engineering, Science and Research Branch, Islamic Azad University, Tehran, and \\ Robotic Research Laboratory, Center of Excellence in Experimental Solid, Mechanics and Dynamics, School of Mechanical \\ Engineering, Iran University of Science and Technology, Tehran, Iran; e-mail: hkorayem@iust.ac.ir
}

\begin{abstract}
This paper aims at planning an optimal point to point path for a flexible manipulator under large deformation. For this purpose, the researchers use a direct method and meta-heuristic optimization process. In this paper, the maximum load carried by the manipulator and the minimum transmission time are taken as objective functions of the optimization process to get optimal path profiles. Kinematic constraints, the maximum velocity and acceleration, the dynamic constraint of the maximum torque applied to the arms and also the constraint of final point accuracy are discussed. For the optimization process, the Harmony Search (HS) method is used. To evaluate the effectiveness of the approach proposed, simulation studies are reviewed by considering a two-link flexible manipulator with the fixed base. The findings indicate that the proposed method is in power of dealing with nonlinear dynamics of the system. Furthermore, the results obtained by rigid, small and large deformation models are compared with each other.
\end{abstract}

Keywords: meta-heuristic optimization, harmony search, large deformation, flexible manipulator

\section{Introduction}

In order to increase the efficiency and economy of manipulators, finding an optimal trajectory by maximum dynamic load capacity and minimum transmission time between two points is of particular and high importance. When planning a point to point path, there are many complexities, so different methods should be used to solve the problem. All used methods are a subset of two main methods, namely direct and indirect methods. In general, the indirect methods are based on the optimal control theory and the minimum principle of Pontryagin. The planning of a two-link rigid manipulator is done via using the optimal control by Korayem et al. (2009). To calculate Dynamic Load Carrying Capacity (DLCC) in a manipulator, the optimal control method is studied through considering complete dynamics of the system and nonlinear terms. Boundary conditions are exactly satisfied and the optimization problem is numerically solved with sufficient accuracy. Korayem and Nikoobin (2009) studied the optimal path planning for rigid and fixed-base two-link manipulators with the help of the optimal control method. By using Pontryagin optimality conditions, the determination of the maximum load capacity will be changed to the standard two-point boundary value problem (Korayem and Nikoobin, 2009). Korayem et al. (2011) planned an optimal path for a two-link mobile manipulator by using the indirect method. They proved that the implementation of the optimal control considering full 
nonlinear dynamics of the system caused by Hamiltonian gradient calculation, the existence of a two-point boundary value problem and the use of a multiple shooting numerical method are not so easy.

In all activities mentioned using the indirect methods and the optimal control theory to plan the optimal path, there are the following limitations. Path planning by the optimal control needs nonlinear numerical techniques such as multiple shooting methods. These techniques require a good initial guess and are sensitive to it. These methods also require an analytical form of the Hamiltonian gradient and an optimum value of state variables obtained by using the Hamiltonian gradient. In these methods, the state variables are used to solve the problem that in the general case (especially by considering the flexibility) it is very difficult to estimate them. Furthermore, precise determination of weight functions for different objective functions will make some problems in the implementation of the methods presented. To resolve the defects, direct approaches are recommended to plan the path. Direct methods are based on discretization of dynamic variables of the system (state variables and control variables) in which ultimately, to provide the parameters, the problem of planning a path will result in a parametric optimization problem. In these methods, the profile of joints motion is represented directly by polynomial, Spline and B-spline functions. By the profile of joints motion, the path planning will be changed to the optimization problem to determine the optimality of profile constant coefficients. To solve the optimization problem, meta-heuristic approaches speeding up the convergence and decreasing the sensitivity to the initial guess can be used. To implement these methods, there is no requirement for linearization and simplification of dynamic equations of the system, and so complete dynamics of the system can be considered.

When planning a trajectory, different functions are considered as objective functions in optimization. The most important ones are the minimum transmission time (Haddad et al., 2005), minimum energy consumption (Spangelo and Egeland, 1992) and minimum jerk in joints (Piazzi and Visioli, 2000). Different methods are developed to study the path planning by the direct method presented such as Genetic Algorithm method (GA) (Garg and Kumar, 2002; Saravanan and Ramabalan, 2008; Saravanan et al., 2008), Simulated Annealing (SA), Sequential Weight Increasing Factor Technique (SWIFT) and Sequential Quadratic Programming (SQP) method (Chettibi et al., 2004). In (Chettibi et al., 2004), SQP method is used to plan the path. It is clear that SQP may get a local optimum, and for convergence of this optimal solution, it is necessary to select the initial guess appropriately. Haddad et al. (2007) studied a point to point path planning problem for a mobile base manipulator. They used the direct method and random optimization to plan the path. The path was generated based on the objective function of minimum transmission time and the kinematic constraints of velocity, acceleration as aell as the dynamic constraint of torque of the motors. Furthermore, the path planning was also done based on the stability constraint of the manipulator by ZMP method. Tangpattanakul and Artrit (2009) paid attention to path planning of the manipulator based on the objective function of minimum transmission time in the point to point case. The optimization problem was solved by considering kinematic constraints using Harmony Search. The simulation results showed that HS method converged faster than SQP method to the optimal solution, and the sensitivity of this method was less toward the initial guess. Tangpttanakul et al. (2010) investigated the problem of point to point path planning by using HS optimization method. The simulation results proved that HS was a better method for solving the problem of robot path planning. Zanotto et al. (2011) considered the minimum transmission time and jerk as the objective function in the optimization problem. Experimental results were also elaborated to compare with the theoretical results. Chettibi and Lemoine (2007) planned a point to point path by using SQP. They offered a single-link manipulator considering full dynamics of the manipulator and the driving motor. To solve the problem of optimization, the objective function of minimum transmission time and electro-mechanic constraints were used. 
This paper includes the following aspects of innovations. An optimal path is designed for a manipulator by considering the most complete nonlinear dynamics, large deformation model and Timoshenko beam model. In previous papers, direct methods were used and the effects of the flexibility with the large deformation model were rarely considered in manipulators. In addition, by using the optimal control method, simplification of non-linear equations is non-avoidable due to the excessive complexity of the solution process. In most papers employing direct methods, the minimum transmission time, minimum energy consumption and minimum jerk are considered as the objective function. However, in this paper, the minimum transfer time besides the maximum load carrying capacity of manipulators is assumed as the objective function. In this paper, the Harmony Search method is used to determine the load carrying capacity of the manipulators that have no restrictions to nonlinear dynamics of the systems. The methods like the optimal control have some difficulties when dealing with such issues. So, here, the most complete process is used to design an optimal path using HS method. The proposed optimization method has the following advantages: (1) a global optimal solution is possible, (2) it is easy to program and implement efficiently, (3) it ensures that the resulting optimized trajectory is smoother, faster, and nonsingular, (4) it can also be extended to get optimized trajectories for other types of robots, (5) it considers both kinematic and dynamic aspects of the robot, (6) it considers the payload constraint, and (7) it is computationally superior and faster. The paper is thus organized in the following Sections. In Section 2, dynamic modeling of the manipulator is investigated in the large deformation case. In Section 3, the problem of path planning is discussed by a direct method. Section 4 deals with the extraction of the optimization problem arising from path planning. The method proposed for solving the optimization problem and a flowchart of optimal path planning are presented in Section 5. Finally, the simulation results are presented for a two-link flexible manipulator.

\section{Dynamic analysis of a flexible manipulator under large deformation}

Consider an $m$-link manipulator (Fig. 1) by n degrees of freedom $(n>m)$ that should move from the initial position $X^{i n i}$ to the final position $X^{\text {fin }}$ in space of end effector (assume $q_{r}^{\text {fin }}, q_{r}^{i n i}$ are corresponding joint variables). For dynamic modeling, arms are based on the Timoshenko beam model, and also shear effects are considered. The movement of the arms will be described by the rotation angle of the links $\theta_{i}$, flexible displacement $w_{i}\left(x_{i}, t\right)$ and rotation caused by the flexible displacement $\psi_{i}$.

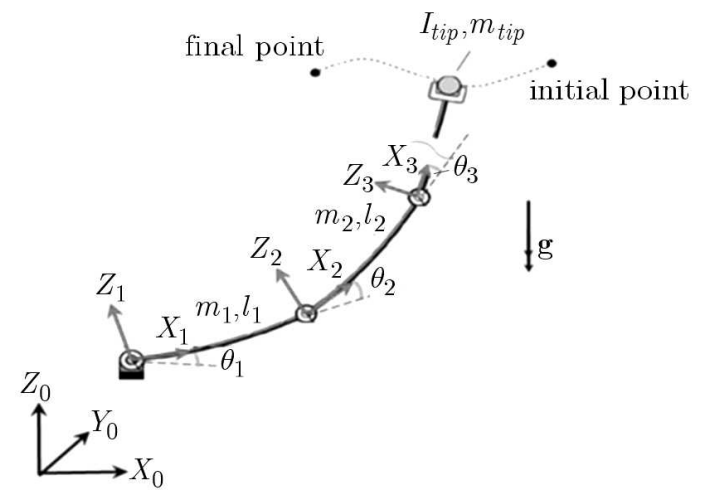

Fig. 1. $m$-link flexible manipulator with a fixed base

The displacement field for the Timoshenko beam with large deformation is as follows

$$
u_{x}=-Z \sin \psi(x, t) \quad u_{z}=w(x, t)+Z \cos \psi(x, t)
$$


where $Z$ is the distance to the neutral axis of the beam. Non-zero elements of the Green strain tensor in the case of large deformation can be written as follows

$$
\begin{aligned}
& E_{11}=E_{0}+Z K_{b}=\underbrace{\frac{1}{2}\left(w_{, x}^{2}\right)}_{E_{0}}+Z \underbrace{\left[-\psi_{, x}\left(\cos \psi+w_{, x} \sin \psi\right)\right]}_{K_{b}} \\
& E_{12}=\frac{1}{2}\left(-\sin \psi+w_{, x} \cos \psi\right)=\Lambda
\end{aligned}
$$

where $E_{0}$ is axial strain, $K_{b}$ - curve occurred in the beam element by large deformation, $\Lambda$ shear strain.

In the above expression, $w_{, x}=\partial w(x, t) / \partial x$. Kinetic energy of the system equals to kinetic energy of the arms $K E_{\text {arm }}$ and tip $K E_{t i p}$ mass

$$
K E=K E_{\text {arm }}+K E_{\text {tip }}
$$

where

$$
\begin{aligned}
& K E_{\text {arm }}=\sum_{i=1}^{m} K E_{i, a r m} \quad K E_{i, a r m}=\frac{1}{2} \int \rho_{i}\left[\dot{\mathbf{p}}_{i}^{\mathrm{T}}\left(x_{i}, t\right) \dot{\mathbf{p}}_{i}\left(x_{i}, t\right)\right] d v_{i} \\
& K E_{\text {tip }}=\frac{1}{2} m_{t i p}\left(\dot{\mathbf{p}}_{\text {tip }}^{\mathrm{T}} \dot{\mathbf{p}}_{t i p}\right)+\frac{1}{2} I_{t i p}\left(\sum_{j=1}^{m} \dot{\theta}_{j}\right)^{2}
\end{aligned}
$$

where $\mathbf{p}_{i}\left(x_{i}, t\right)$ is the position of a small element on the $i$-th flexible arm and $\mathbf{p}_{t i p}$ is the position vector of tip mass with respect to the absolute coordinates $\left\{X_{0}, Y_{0}, Z_{0}\right\}$ defined as follows

$$
\begin{aligned}
& \mathbf{p}_{i}\left(x_{i}, t\right)=\sum_{j=0}^{i-1} \mathbf{B}_{j}\left(l_{j}, t\right)+\mathbf{B}_{i}\left(x_{i}, t\right) \\
& \mathbf{B}_{i}\left(x_{i}, t\right)=\left[x_{i} \cos \left(\theta_{i-1}+\theta_{i}\right)-w_{i}\left(x_{i}, t\right) \sin \left(\theta_{i-1}+\theta_{i}\right)\right] \mathbf{I} \\
& \quad+\left[x_{i} \sin \left(\theta_{i-1}+\theta_{i}\right)+w_{i}\left(x_{i}, t\right) \cos \left(\theta_{i-1}+\theta_{i}\right)\right] \mathbf{K} \\
& \mathbf{p}_{\text {tip }}=\sum_{j=1}^{m} \mathbf{B}_{j}\left(l_{j}, t\right) \quad(i=1,2, \ldots, m) \quad \mathbf{B}_{0}=\mathbf{0} \quad \theta_{0}=0
\end{aligned}
$$

Potential energy of the system consists of strain energy and gravitational potential energy

$$
\begin{array}{rlr}
P E & =\frac{1}{2} \sum_{i=1}^{m} \int_{0}^{l_{i}}\left(E_{i} A_{i} E_{i, 0}^{2}+E_{i} I_{i} K_{i, b}^{2}+k G_{i} A_{i} \Lambda_{i}^{2}\right) d x_{i} \\
& +\sum_{i=1}^{m} \rho_{i} A_{i} g\left(\sum_{j=0}^{i-1} H_{j}\left(l_{j}, t\right)+\int_{0}^{l_{i}} H_{i}\left(x_{i}, t\right) d x_{i}\right)+m_{t i p} g \sum_{j=1}^{m} H_{j}\left(l_{j}, t\right) \\
H_{i}\left(x_{i}, t\right)=x_{i} \sin \left(\theta_{i-1}+\theta_{i}\right)+w_{i}\left(x_{i}, t\right) \cos \left(\theta_{i-1}+\theta_{i}\right) \quad H_{0}=0 & \theta_{0}=0
\end{array}
$$

where $g$ is the acceleration of gravity.

\section{Problem statement}

By separating the joint and flexibility variables and applying the extended Hamilton method, the general form of the $m$-link manipulator equations is as follows

$$
\left[\begin{array}{ll}
\mathbf{M}_{f} & \mathbf{M}_{r}
\end{array}\right]\left[\begin{array}{c}
\ddot{\mathbf{q}}_{f} \\
\ddot{\mathbf{q}}_{r}
\end{array}\right]+\left[\begin{array}{l}
\mathbf{C}_{f}(\mathbf{q}, \dot{\mathbf{q}}) \\
\mathbf{C}_{r}(\mathbf{q}, \dot{\mathbf{q}})
\end{array}\right]+\left[\begin{array}{l}
\mathbf{Q}_{f}(\mathbf{q}) \\
\mathbf{Q}_{r}(\mathbf{q})
\end{array}\right]=\left[\begin{array}{c}
\mathbf{0} \\
\boldsymbol{\tau}
\end{array}\right]
$$


where $\mathbf{M}_{f}$ is the inertia matrix for flexibility degrees of freedom in the manipulator, $\mathbf{M}_{r}-$ inertia matrix for rigid degrees of freedom, the vector $\mathbf{q}$ contains generalized coordinates, $\mathbf{q}_{r}, \mathbf{q}_{f}$ are vectors of the flexibility coordinates and joint coordinates, respectively, $\mathbf{C}_{f}(\mathbf{q}, \dot{\mathbf{q}}), \mathbf{C}_{r}(\mathbf{q}, \dot{\mathbf{q}})-$ Coriolis and centrifugal force vector for flexible and for rigid degrees of freedom, $\mathbf{Q}_{f}(\mathbf{q}), \mathbf{Q}_{r}(\mathbf{q})$ - gravity vector for flexible and for rigid degrees of freedom, $\boldsymbol{\tau}$ - torque applied to the joints. By having both initial and final points of the end effector, the main purpose is to determine the maximum load capacity, transmission time, joint variables vector, the corresponding velocity and torque vector applied to the manipulator so that to satisfy all the constraints in the system. To solve the problem by thr direct method, the profile of the joints path will be approximated directly through interpolation functions such as polynomial, Spline and B-Spline functions, and the problem of path planning will be changed to a parametric optimization problem in order to calculate the constants of interpolation functions. Then, the optimization problem can be solved by using meta-heuristic optimization.

\subsection{Optimization by the objective function of maximum load carrying capacity}

In this case, the objective of path planning in the point to point case is to calculate a trajectory for the robot end effector in which the manipulator can carry the maximum dynamic load and the kinematic constraints of the joints, velocity and acceleration, torque dynamic constraint and end point accuracy constraint will be satisfied. In addition, the optimal path must be planned not to meet manipulator singularity configurations. The problem of planning an optimal path by considering the objective function and above the constraints will be presented in the form of an optimization problem as follows

$$
\begin{aligned}
& f_{\text {obj }}=\max \left(m_{\text {tip }}, I_{\text {tip }}\right) \\
& \text { subject to }(i=1,2, \ldots, m)\left\{\begin{array}{ll}
\left|q_{r, i}(t)\right| \leqslant q_{r, i}^{\max } & \left|\dot{q}_{r, i}(t)\right| \leqslant k_{v i} \\
\left|\tau_{i}(t)\right| \leqslant \tau_{i}^{\text {max }} & \left|q_{r, i}^{\text {fin }}-q_{r, i}(T)\right| \leqslant \varepsilon
\end{array} \quad\left|\ddot{q}_{r, i}(t)\right| \leqslant k_{a i}\right.
\end{aligned}
$$

where $m_{t i p}, I_{t i p}$ are the concentrated mass and moment inertia of the end manipulator, $\tau_{i}^{\max }-$ maximum torque applied to the joints.

\subsection{Optimization by the objective function of minimum transmission time}

In this case, minimization of the transmission time between the initial and final points is the aim of optimal path planning. Assume $\widetilde{q}_{r}(t)$ as a candidate selected for the profile of the joints path. By using a change in the variable $\zeta=t / T(0 \leqslant \zeta \leqslant 1)$, any kinematic and dynamic constraints presented in the previous Section are discussed as bands of the transmission time. The constraints governing joint velocity and acceleration can be converted into the following formulas by applying the derivation chain rule

$$
\begin{array}{ll}
T \geqslant T_{v} \Rightarrow T_{v}=\max _{i=1,2, \ldots, m}\left[\max _{\zeta \in[0,1]} \frac{\left|\widetilde{q}_{r, i}^{\prime}(\zeta)\right|}{k_{v i}}\right] & \widetilde{q}_{r, i}^{\prime}(\zeta)=\frac{d \widetilde{q}_{r, i}(\zeta)}{d \zeta} \\
T \geqslant T_{A} \Rightarrow T_{A}=\max _{i=1,2, \ldots, m}\left[\max _{\zeta \in[0,1]} \frac{\left|\widetilde{q}_{r, i}^{\prime \prime}(\zeta)\right|}{k_{a i}}\right]^{\frac{1}{2}} & \widetilde{q}_{r, i}^{\prime \prime}(\zeta)=\frac{d^{2} \widetilde{q}_{r, i}(\zeta)}{d \zeta^{2}}
\end{array}
$$

Dynamic constraints determination the motors torque may be changed to constraints of two bands in terms of the transmission time so that $T \in\left[T_{L}, T_{R}\right]$. By examining the bands time obtained, finally, the end band $\left[T_{\text {lower }}, T_{\text {upper }}\right]$ will be find for the transmission time. The optimal transmission time for each profile of the trajectory can be obtained by minimizing the objective function based on the transmission time of the period. By using a change in the variable $\zeta=t / T$, 
the differential equation of motion for the $i$-th variable of the joint in the $m$-link manipulator will be as below

$$
\begin{array}{ll}
\bar{\tau}_{i}(\zeta)=\frac{1}{T^{2}} \bar{h}_{r, i}(\zeta)+\bar{Q}_{r, i(\zeta)} \quad \zeta \in[0,1] & i=1,2, \ldots, m \\
h_{r, i}(\zeta)=\sum_{j=1}^{m} M_{r, i j} \widetilde{q}_{r, j}^{\prime \prime}(\zeta)+C_{r, i}\left(\widetilde{q}(\zeta), \widetilde{q}^{\prime}(\zeta)\right) & \\
\bar{h}_{r, i}(\zeta)=\frac{h_{r, i}(\zeta)}{\tau_{i}^{\text {max }}} \quad \bar{Q}_{r, i}(\zeta)=\frac{Q_{r, i}(\widetilde{q}(\zeta))}{\tau_{i}^{\text {max }}} & \bar{\tau}_{i}(\zeta)=\frac{\tau_{i}(\zeta)}{\tau_{i}^{\text {max }}}
\end{array}
$$

So, the dynamic constraints will be changed to the following equation

$$
\begin{array}{ll}
-1 \leqslant \frac{1}{T^{2}} \bar{h}_{r, i}(\zeta)+\bar{Q}_{r, i(\zeta)} \leqslant 1 & -b_{i}(\zeta) \leqslant \frac{1}{T^{2}} \bar{h}_{r, i}(\zeta) \leqslant a_{i}(\zeta) \\
a_{i}(\zeta)=1-\bar{Q}_{r, i(\zeta)} & b_{i}(\zeta)=1+\bar{Q}_{r, i(\zeta)}
\end{array}
$$

Thus, for any $\zeta \in[0,1]$, the time bands $T$ related to the torque constraint are given in Table 1 .

Table 1. Transmission time bands of the path profile selected for dynamic constraints

\begin{tabular}{|c|c|c|c|c|c|}
\hline \multicolumn{2}{|c|}{$\bar{h}_{r, i}(\zeta) \geqslant 0$} & \multicolumn{3}{|c|}{$\bar{h}_{r, i}(\zeta)<0$} \\
\hline \multirow{2}{*}{$a_{i}(\zeta)<0$} & $a_{i}(\zeta) \geqslant 0$ & $b_{i}(\zeta)<0$ & \multicolumn{2}{|c|}{$b_{i}(\zeta) \geqslant 0$} \\
\hline \hline$\emptyset$ & $b_{i}(\zeta)<0$ & $b_{i}(\zeta) \geqslant 0$ & & $a_{i}(\zeta)<0$ & $a_{i}(\zeta) \geqslant 0$ \\
\cline { 2 - 3 } & $T_{L, i}=\sqrt{\frac{\bar{h}_{r, i}(\zeta)}{a_{i}}}$ & $T_{L, i}=\sqrt{\frac{\bar{h}_{r, i}(\zeta)}{a_{i}}}$ & $\emptyset$ & $T_{L, i}=\sqrt{\frac{\bar{h}_{r, i}(\zeta)}{a_{i}}}$ & $T_{L, i}=\sqrt{\frac{\bar{h}_{r, i}(\zeta)}{-b_{i}}}$ \\
& $T_{R, i}=\sqrt{\frac{\bar{h}_{r, i}(\zeta)}{-b_{i}}}$ & $T_{R, i} \rightarrow+\infty$ & & $T_{R, i}=\sqrt{\frac{\bar{h}_{r, i}(\zeta)}{-b_{i}}}$ & $T_{R, i} \rightarrow+\infty$ \\
\hline
\end{tabular}

In general, the problem of optimal path planning by objective function of minimum transmission time and the presented constraints are developed in the form of the optimization problem

$$
\begin{aligned}
& f_{o b j}=\min (T) \\
& \text { subject to } \begin{cases}T \geqslant T_{v} & T_{v}=\max _{i=1,2, \ldots, m}\left[\max _{\zeta \in[0,1]} \frac{\left|\widetilde{q}_{r, i}^{\prime}(\zeta)\right|}{k_{v i}}\right] \\
T \geqslant T_{A} & T_{A}=\max _{i=1,2, \ldots, m}\left[\max _{\zeta \in[0,1]} \frac{\left|\widetilde{q}_{r, i}^{\prime \prime}(\zeta)\right|}{k_{a i}}\right]^{\frac{1}{2}} \\
T_{L} \leqslant T \leqslant T_{R} & \text { Table 1 } \\
\left|\widetilde{q}_{r, i}^{f i n}-\widetilde{q}_{r, i}(T)\right| \leqslant \varepsilon & \end{cases}
\end{aligned}
$$

\section{The method of solving the optimization problem}

To solve the problem of optimization, HS meta-heuristic method will be used. For designing the end effector trajectory, it is necessary to model the path profile for any joint by using the profile of a smooth trapezoid or a cubic Spline. For example, the overall form of a smooth trapezoidal profile is always as follows 


$$
\widetilde{q}_{r, j}= \begin{cases}q_{r, j}^{i n i}+D_{j} \frac{1}{1+\zeta_{b}-\zeta_{a}}\left(\frac{2 \zeta^{3}}{\zeta_{a}^{2}}-\frac{\zeta^{4}}{\zeta_{a}^{3}}\right) & 0 \leqslant \zeta<\zeta_{a} \\ q_{r, j}^{i n i}+D_{j} \frac{2 \zeta-\zeta_{a}}{1+\zeta_{b}-\zeta_{a}} & \zeta_{a} \leqslant \zeta<\zeta_{b} \\ q_{r, j}^{i n i}+D_{j}\left[1-\frac{1}{1+\zeta_{b}-\zeta_{a}}\left(\frac{2(1-\zeta)^{3}}{\left(1-\zeta_{b}\right)^{2}}-\frac{(1-\zeta)^{4}}{\left(1-\zeta_{b}\right)^{3}}\right)\right] & \zeta_{b} \leqslant \zeta \leqslant 1\end{cases}
$$

where

$$
D_{j}=q_{r, j}^{f i n}-q_{r, j}^{i n i}
$$

Therefore, by the initial and final points of the trajectory and optimal determination of the variables $\left(\zeta_{b}, \zeta_{a}\right)$ (by observing the condition $0 \leqslant \zeta_{a}, \zeta_{b} \leqslant 1$ ), the optimal form of joint motion will be determined. A flowchart of optimal path planning is represented in Fig. 2 with the help of Harmony Search for the $m$-link manipulator.

\section{Numerical simulation}

Generalized coordinates of a two-link flexible manipulator with a fixed base is considered as the vector $\mathbf{q}=\left[\theta_{1}, \theta_{2}, w_{1}, \psi_{1}, w_{2}, \psi_{2}\right] . w_{1}$ and $w_{2}$ are transverse vibration variables of the first and second arm, respectively. Also, $\psi_{1}$ and $\psi_{2}$ are rotations caused by transverse vibrations of the first and second arm. By using the finite element method and dividing the arms into multiple elements, the flexibility variables of the $i$-th element of the first arm and the flexibility variables of the $j$-th element of the second arm are presented as follows

$$
\begin{array}{llc}
w_{1 i}=\left[N_{1}(x), N_{2}(x)\right]\left\{\widetilde{w}_{1 i}(t)\right\} & \psi_{1 i}=\left[N_{1}(x), N_{2}(x)\right]\left\{\widetilde{\psi}_{1 i}(t)\right\} & i=1,2, \ldots, n_{1} \\
w_{2 j}=\left[N_{1}(x), N_{2}(x)\right]\left\{\widetilde{w}_{2 j}(t)\right\} & \psi_{2 j}=\left[N_{1}(x), N_{2}(x)\right]\left\{\widetilde{\psi}_{2 j}(t)\right\} & j=1,2, \ldots, n_{2} \\
{\left[N_{1}(x), N_{2}(x)\right]=\left[1-\frac{x}{l}, \frac{x}{l}\right]} & &
\end{array}
$$

where $n_{1}$ and $n_{2}$ are equal to the nodes of the elements network of the first and second arm. So, the closed form of dynamic equations of the flexible two-link manipulator is as follows

$$
\begin{aligned}
& {\left[\begin{array}{cccccc}
m_{11} & m_{12} & m_{13} & m_{14} & m_{15} & m_{16} \\
& m_{22} & m_{23} & m_{24} & m_{25} & m_{26} \\
& & m_{33} & m_{34} & m_{35} & m_{36} \\
& & & m_{44} & m_{45} & m_{46} \\
& \text { sym } & & m_{55} & m_{56} \\
i=1,2, \ldots, n_{1} & j=1,2, \ldots, n_{2}
\end{array}\right]\left[\begin{array}{c}
\ddot{\theta}_{1} \\
\ddot{\theta}_{2} \\
\ddot{\ddot{w}}_{1 i} \\
\ddot{\ddot{\psi}}_{1 i} \\
\ddot{\tilde{w}}_{2 j} \\
\ddot{\ddot{\psi}}_{2 j}
\end{array}\right]+\left[\begin{array}{c}
C_{1} \\
C_{2} \\
C_{3 i} \\
0 \\
C_{5 j} \\
0
\end{array}\right]+\left[\begin{array}{c}
Q_{1} \\
Q_{2} \\
Q_{3 i} \\
Q_{4 i} \\
Q_{5 j} \\
Q_{6 j}
\end{array}\right]+\left[\begin{array}{c}
D_{1} \\
D_{2} \\
D_{3 i} \\
D_{4 i} \\
D_{5 j} \\
D_{6 j}
\end{array}\right]=\left[\begin{array}{c}
\tau_{1} \\
\tau_{2} \\
0 \\
0 \\
0 \\
0
\end{array}\right]}
\end{aligned}
$$

where the vector $\mathbf{Q}$ represents the effects of gravitational and potential energy of the system. The vector $\mathbf{D}$ is a disturbance torque or a force vector. It should be noted that $\mathbf{D}$ has different meanings. For example, it can be a friction and reaction torque or force. The end effector position can be offered by the following equation

$$
X_{e}=\left[l_{1} \cos \theta_{1}+l_{2} \cos \left(\theta_{1}+\theta_{2}\right)\right] \mathbf{i}+\left[l_{1} \sin \theta_{1}+l_{2} \sin \left(\theta_{1}+\theta_{2}\right)\right] \mathbf{j}
$$

To optimally plan a path for a two-link manipulator in the point to point case, the optimization problems should be solved as follows: 


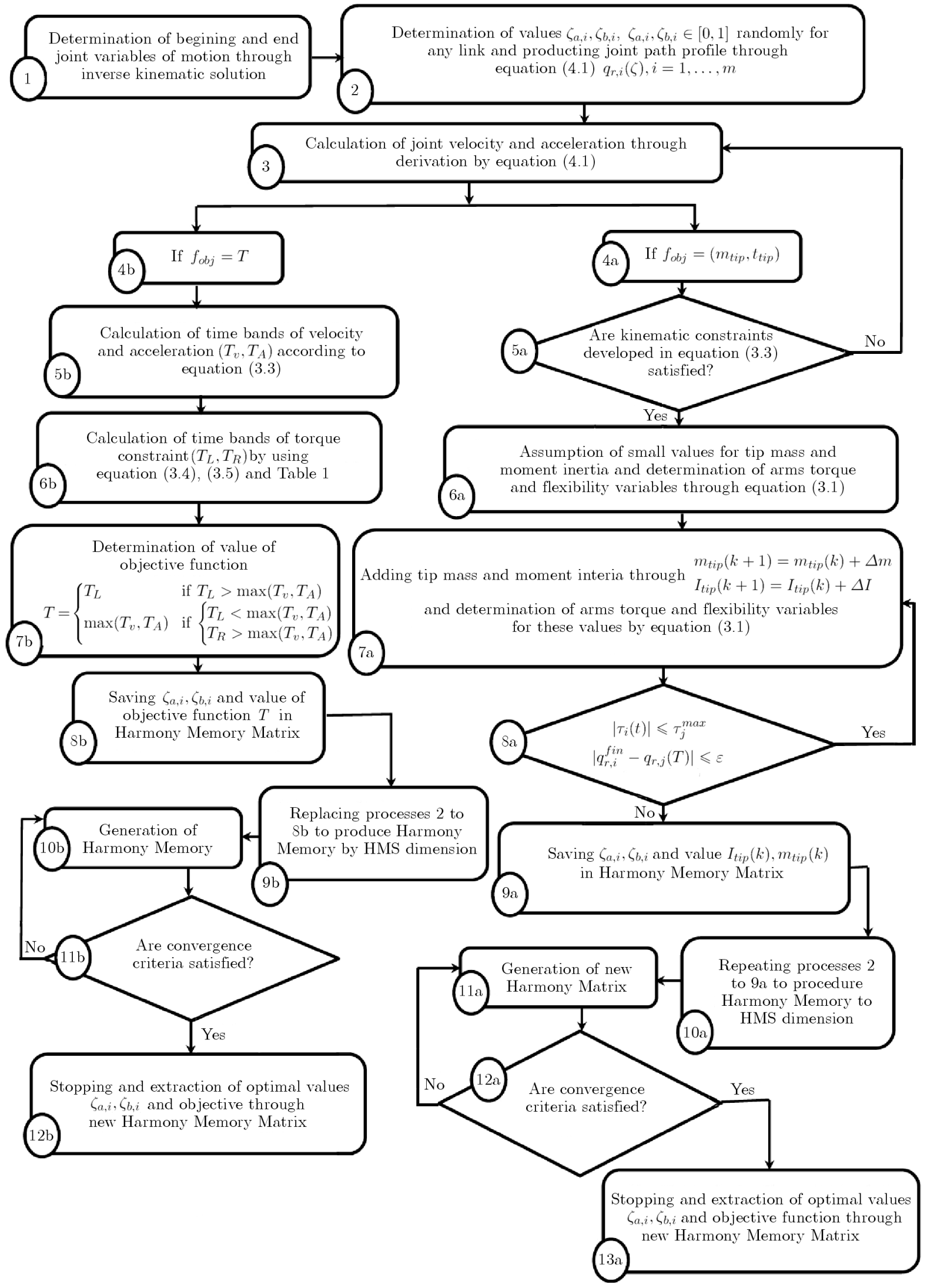

Fig. 2. Optimal path planning flowchart by Harmony Search method 
- Path planning based on the minimum transmission time

$$
\begin{aligned}
& f_{o b j}=\min (T) \\
& \text { design variables }=\left(\zeta_{a 1}, \zeta_{b 1}, \zeta_{a 2}, \zeta_{b 2}\right) \\
& \theta_{i}(t)=\widetilde{q}_{r, i} \quad \text { from Eq. }(4.2) \\
& {\left[\begin{array}{l}
\tau_{1} \\
\tau_{2}
\end{array}\right]=\left[\begin{array}{lll}
m_{11} & \cdots & m_{16} \\
m_{21} & \cdots & m_{26}
\end{array}\right]\left[\begin{array}{l}
\ddot{\theta}_{1} \\
\ddot{\theta}_{2}
\end{array}\right]+\left[\begin{array}{l}
C_{1} \\
C_{2}
\end{array}\right]+\left[\begin{array}{l}
Q_{1} \\
Q_{2}
\end{array}\right] \quad \text { from Eq. }(5.2)} \\
& \text { subject to } \begin{cases}T \geqslant T_{v} & T_{v}=\max _{i=1,2}\left[\max _{\zeta \in[0,1]} \frac{\left|\widetilde{q_{r, i}^{\prime}}(\zeta)\right|}{k_{v i}}\right] \\
T \geqslant T_{A} & \max _{i=1,2}\left[\max _{\zeta \in[0,1]} \frac{\left|\widetilde{q}_{r, i}^{\prime \prime}(\zeta)\right|}{k_{a i}}\right]^{\frac{1}{2}} \\
T_{L} \leqslant T \leqslant T_{R} & \text { from Eq. }(3.6) \text { and Table } 1 \\
\left|X_{e}(T)-X^{f i n}\right| \leqslant \varepsilon & \end{cases}
\end{aligned}
$$

- Path planning based on the maximum load capacity

$$
\begin{aligned}
& f_{o b j}=\max \left(m_{t i p}\right) \\
& \text { design variables }=\left(\zeta_{a 1}, \zeta_{b 1}, \zeta_{a 2}, \zeta_{b 2}\right) \\
& \theta_{i}(t)=\widetilde{q}_{r, i} \quad \text { from Eq. }(4.2) \\
& {\left[\begin{array}{l}
\tau_{1} \\
\tau_{2}
\end{array}\right]=\left[\begin{array}{lll}
m_{11} & \cdots & m_{16} \\
m_{21} & \cdots & m_{26}
\end{array}\right]\left[\begin{array}{l}
\ddot{\theta}_{1} \\
\ddot{\theta}_{2}
\end{array}\right]+\left[\begin{array}{l}
C_{1} \\
C_{2}
\end{array}\right]+\left[\begin{array}{l}
Q_{1} \\
Q_{2}
\end{array}\right] \quad \text { from Eq. }(5.2)} \\
& \text { subject to }\left\{\begin{array}{l}
\left|\theta_{i}(t)\right| \leqslant \theta_{i}^{\text {max }} \\
\left|\dot{\theta}_{i}(t)\right| \leqslant k_{v i} \\
\left|\ddot{\theta}_{i}(t)\right| \leqslant k_{a i} \\
\left|\tau_{i}(t)\right| \leqslant \tau_{i}^{\text {max }} \\
\left|X_{e}(T)-X^{\text {fin }}\right| \leqslant \varepsilon
\end{array} \quad i=1,2\right.
\end{aligned}
$$

To validate the results, simulation of a planar two-link manipulator described by Heidari (2011) will be done and compared with the results obtained by the optimal control method. Table 2 shows the parameters of the manipulator assumed.

Table 2. Parameters of the flexible two-link manipulator (Heidari, 2011)

\begin{tabular}{|l|c|l|c|}
\hline \multicolumn{1}{|c|}{ Parameter (unit) } & Value & \multicolumn{1}{|c|}{ Parameter (unit) } & Value \\
\hline \hline Length of links $[\mathrm{m}]$ & $l_{1}=l_{2}=0.5$ & Moment of inertia $\left[\mathrm{m}^{4}\right]$ & $I_{1}=I_{2}=2.5 \cdot 10^{-9}$ \\
\hline Mass $[\mathrm{kg}]$ & $m_{1}=m_{2}=3$ & $\begin{array}{l}\text { Young's modulus of } \\
\text { material }\left[\mathrm{N} / \mathrm{m}^{2}\right]\end{array}$ & $E_{1}=E_{2}=2 \cdot 10^{10}$ \\
\hline
\end{tabular}

The initial position of the end effectors, when $t=0$, is at the point $(0.5,0)$ and when $t=1 \mathrm{~s}$, is at the point $(0.5,0.5)$. The end effector velocity at the beginning and end of the path is also assumed to be zero. To get rid of the singularity case in the manipulator, the constraint $\theta_{2}(t) \neq 0^{\circ}$ and $180^{\circ}$ is considered in the optimization problem. The maximum torque of the motors is equal to $\tau_{\max }=8 \mathrm{Nm}$. The results of joints torque and the path paved by the end effector by considering the minimum of torque as the objective function for three cases including rigid arms, arm with small deformation and an arm with large deformation are shown in Figs.3 and 4. Figures 3a,b indicate that by taking the effect of flexibility of the arms into account, the torques resulting from the large and small deformation exhibit fluctuation behavior to the rigidity, and the torque resulting from large deformation is slightly greater than that from small deformation. Figure 4 indicates that the paths obtained by small and large deformation models 
do not reach the end point and deviation occurs. The deviation at the start point is large, and the fluctuations of the end effector path are increased by greater flexibility. The results obtained are in good agreement with the results reported by Heidari (2011).
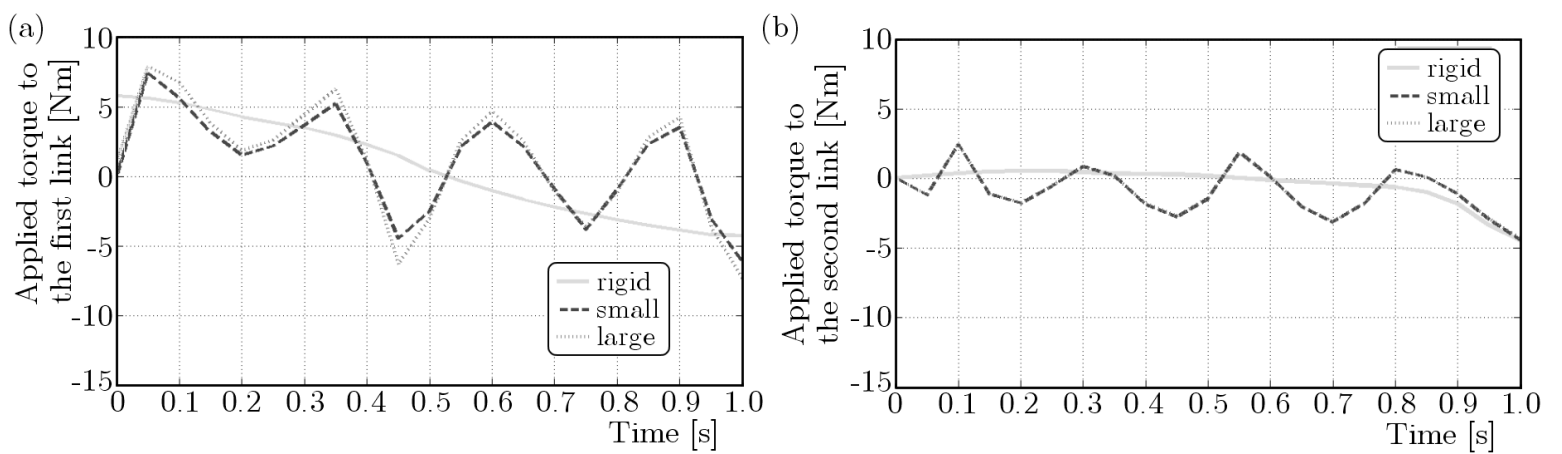

Fig. 3. Torque applied to the (a) first link and (b) second link

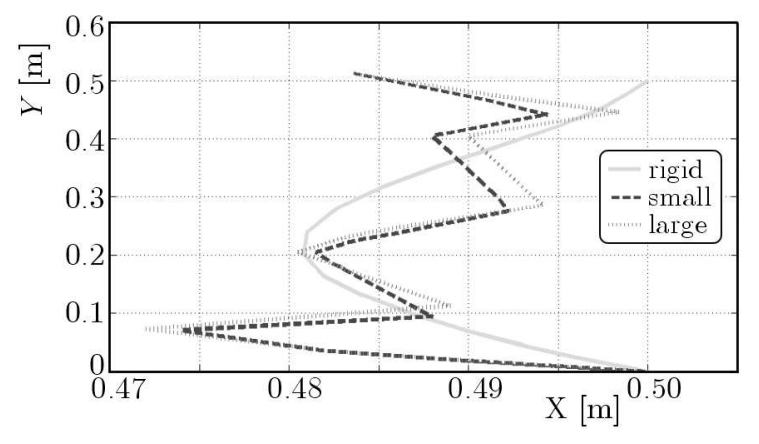

Fig. 4. End effector trajectory for the planar two-link manipulator

\subsection{Results of path planning by the maximum load carrying capacity}

This Section addresses the planning of an optimal path by considering the maximum load capacity as the objective function. For this purpose, a two-link manipulator is taken on the plane $X Z$ in which the end effector moves from $x_{1}=1.6 \mathrm{~m}, z_{1}=0 \mathrm{~m}$ and after the time $t=2 \mathrm{~s}$ stops in the point $x_{2}=1.2 \mathrm{~m}, z_{2}=1.2 \mathrm{~m}$. For HS optimization method, harmony memory size also is $\mathrm{HMS}=10$, the harmony memory considering rate is $\mathrm{HMCR}=0.75$ and the pitch adjustment rate is $\mathrm{PAR}=0.25$. The maximum velocity and acceleration equal to $k_{v}=3 \mathrm{rad} / \mathrm{s}, k_{a}=10 \mathrm{rad} / \mathrm{s}^{2}$ respectively, and the maximum torque is $\tau_{\max }=230 \mathrm{Nm}$. The allowed error from the final point is $\varepsilon=2 \mathrm{~cm}$ as well. Manipulator parameters specified in this Section is are given in Table 3. In all following figures, a smooth trapezoidal profile is considered as profile 1 and a cubic Spline profile as profile 2. By solving inverse kinematics, the values of position and joints velocity at the beginning and end of the path are obtained as follows

$$
\begin{array}{ll}
\theta_{1}(0)=-1.4455 \mathrm{rad} & \theta_{2}(0)=-0.4240 \mathrm{rad} \\
\theta_{1}(2)=1.6961 \mathrm{rad} & \theta_{2}(2)=1.4455 \mathrm{rad} \\
\dot{\theta}_{1}(0)=\dot{\theta}_{2}(0)=\dot{\theta}_{1}(2)=\dot{\theta}_{2}(2)=0 &
\end{array}
$$

The results of these three cases including rigid arms, arms with small deformation and arms with large deformation are presented. The results of the maximum load by considering kinematic and dynamic constraints for both profiles are presented in Table 4. By studying the figures related to the angular displacement and arms torque, it is clear that wherever the slope of torque figure is greater, the corresponding angular displacements will change more quickly, and any change 
Table 3. Parameters of the two-flexible link manipulator

\begin{tabular}{|l|c|l|c|}
\hline Parameter & Value (unit) & Parameter & Value (unit) \\
\hline \hline Length of links & $l_{1}=0.4 \mathrm{~m}, l_{2}=1.6 \mathrm{~m}$ & Cross-section area & $A_{1}=A_{2}=2.5 \cdot 10^{-3} \mathrm{~m}^{2}$ \\
Density of links & $\rho_{1}=\rho_{2}=3000 \mathrm{~kg} / \mathrm{m}^{3}$ & Moment of inertia & $I_{1}=I_{2}=5.2 \cdot 10^{-7} \mathrm{~m}^{4}$ \\
Young's modulus & $E_{1}=E_{2}=$ & Shear modulus & $G_{1}=G_{2}=16 \cdot 10^{5} \mathrm{~N} / \mathrm{m}^{2}$ \\
of material & $0.3 \cdot 10^{11} \mathrm{~N} / \mathrm{m}^{2}$ & & \\
\hline
\end{tabular}
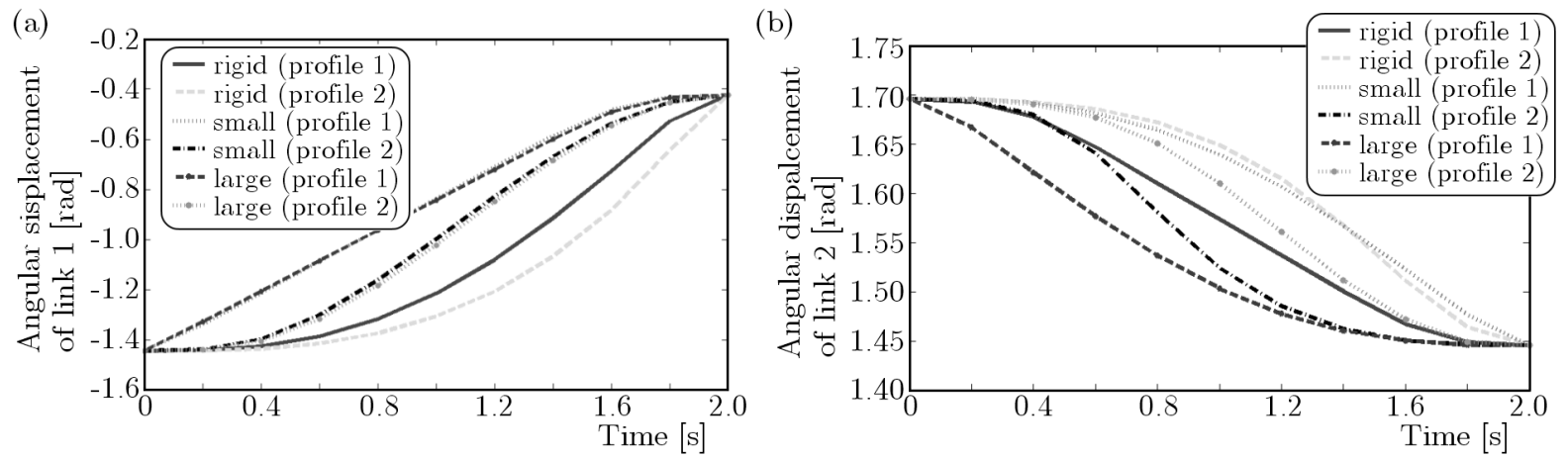

Fig. 5. Angular displacement of the (a) first link and (b) second link
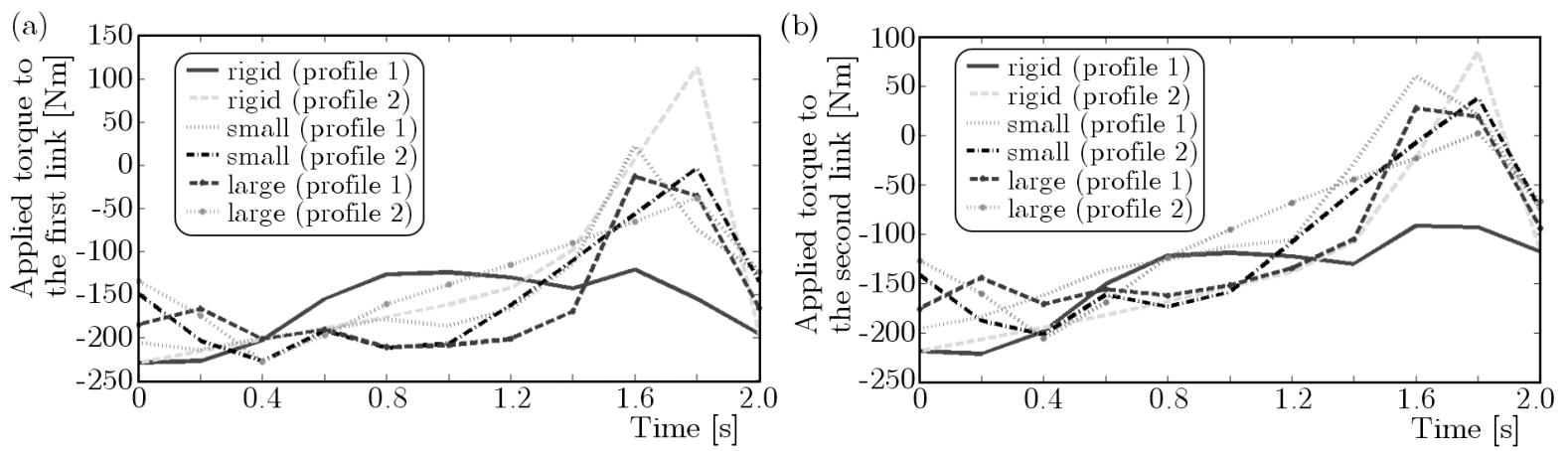

Fig. 6. Torque applied to the (a) first link and (b) second link

in the torque direction will alter the angular displacement of the arms. Figure 7 indicates that the path obtained for profiles in different cases are smooth. By studying the figures and the results obtained in this Section, it can be seen that the flexibility of the arms has a significant effect on the problem of manipulator path planning so that the load capacity of the manipulator for both profiles is less than the rigid one by taking into account the flexibility of the arms. This difference is due to the limitation of the engine torque caused by torque fluctuations in the small and large deformation models. A more careful flexibility analysis of the model developed to be carried out and more complete nonlinear terms to be considered makes the results for the load carrying capacity to decrease. Therefore, small deformation is no longer a complete model for studying the effects of flexibility. Harmony Search method is appropriately consistent with nonlinear dynamics of the system and for implementing this method. There is no need to simplify the dynamic equations of the system. The results indicate superiority of the smooth trapezoidal profile over the cubic Spline profile. Since, the load carrying capacity for the smooth trapezoidal profile is greater than that for the cubic Spline profile. According to the results obtained, it can be said as far as the capabilities of the method proposed are concerned that this method has no problem with nonlinear dynamics of the manipulator, and the optimal path will be obtained with appropriate convergence speed. It has potential to consider all kinematic, dynamic, and singularity constraints as well as the end point accuracy constraint at the same time in the optimization process. 
Table 4. Results of optimally path planning by the maximum load capacity

\begin{tabular}{|c|c|c|}
\hline Profile & Model & DLCC $[\mathrm{kg}]$ \\
\hline \hline \multirow{2}{*}{$\begin{array}{c}\text { Smooth } \\
\text { trapezoidal }\end{array}$} & rigid & 8.4 \\
\cline { 2 - 3 } & small & 6.9 \\
\cline { 2 - 3 } & large & 5.6 \\
\hline \multirow{2}{*}{$\begin{array}{c}\text { Cubic } \\
\text { spline }\end{array}$} & rigid & 8.4 \\
\cline { 2 - 3 } & small & 3.3 \\
\cline { 2 - 3 } & large & 2.4 \\
\hline
\end{tabular}

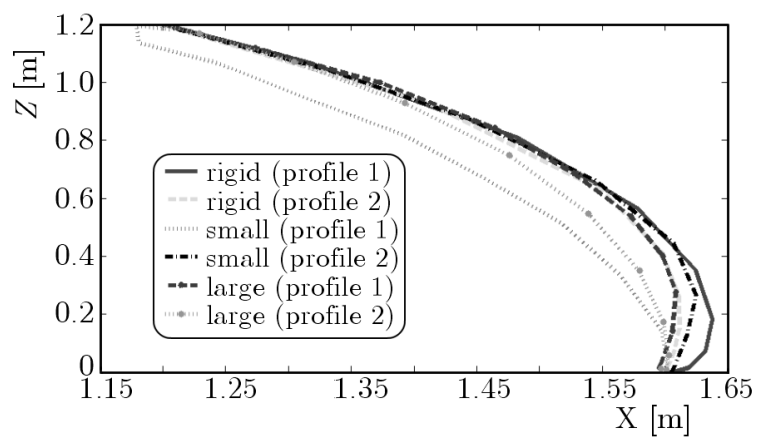

Fig. 7. The end effector trajectory in rigid, small and large deformation models

\subsection{The results of planning the optimal path by minimum transmission time}

The planning of an optimal path will be investigated here by considering the minimum transmission time as the objective function. The results are presented in form of a figure for the cubic Spline profile only. By reviewing the previous Section and taking $m_{t i p}=1.5 \mathrm{~kg}$, the results of path planning are presented in Figs. 8 and 9 for arms corresponding to the large deformation model. Since, the angular displacement of the first arm during motion is larger than the angular displacement of the second arm, as shown in Fig. 8b, the angular velocity of the first arm is greater than that of the second arm. Similarly, the torque applied to the first arm is greater than

(a)

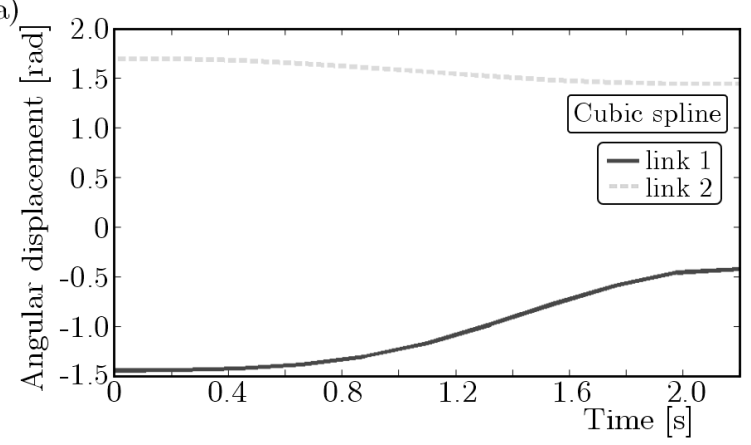

(b)

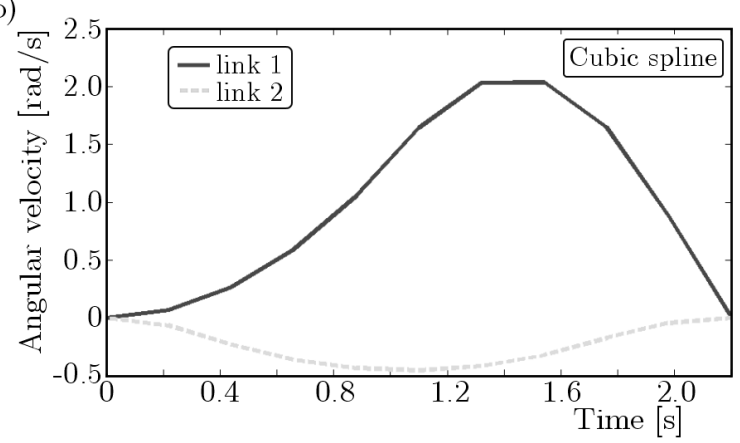

Fig. 8. Angular displacement (a) and angular velocity (b) of the first and second links in the large deformation model

that in the second arm, and the engine of the first arm will reach its saturation point sooner. The optimal path planned for all three models are shown in Fig. 10. By studying this figure, it is clear that the path planned for the small and large deformation models does not reach the end point, and there is a distance equal to the allowed amount $\varepsilon=2 \mathrm{~cm}$. The results of the transmission time by considering kinematic and dynamic constraints and HS method for both profiles are given in Table 5. It shows that HS method is efficient enough to solve the optimal robot trajectory planning. What is presented in the table indicates that the flexibility of the 
arms increases the time of transmission for both profiles. The transmission times obtained for the manipulator through the rigid and small deformation models are not much different. But, the value of this parameter in the large deformation model is greater than that in rigid and small deformation models. In this case, the results obtained from the smooth trapezoidal profile are even better than the results of the cubic Spline profile.

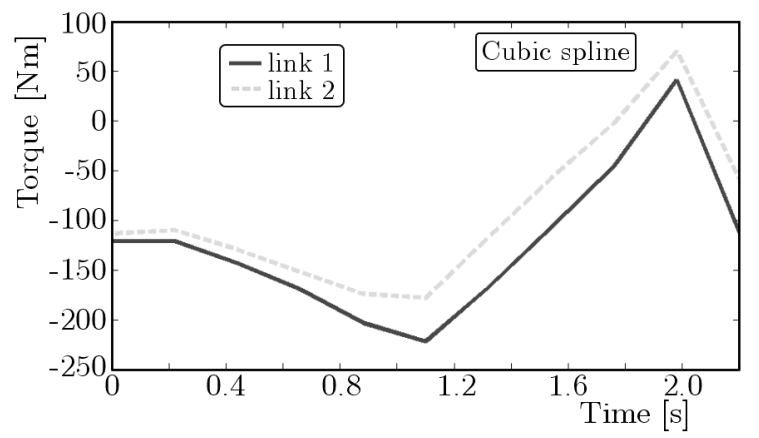

Fig. 9. Torque applied to the first and second links in the large deformation model

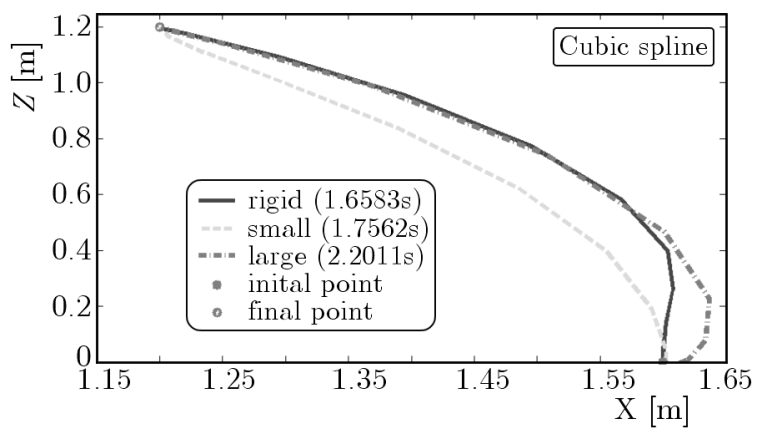

Fig. 10. Optimal path paved by the end effector for the minimum transmission time in rigid, small and large deformation models

Table 5. Results of the optimal path planning by the minimum transmission time

\begin{tabular}{|c|c|c|}
\hline \multirow{2}{*}{ Profile } & Model & $\begin{array}{c}\text { Minimum transmission } \\
\text { time }[\mathrm{s}]\end{array}$ \\
\hline \hline \multirow{2}{*}{$\begin{array}{l}\text { Smooth } \\
\text { trapezoidal }\end{array}$} & rigid & 0.8794 \\
\cline { 2 - 3 } & small & 0.9152 \\
\cline { 2 - 3 } & large & 1.5603 \\
\hline \multirow{2}{*}{$\begin{array}{c}\text { Cubic } \\
\text { spline }\end{array}$} & rigid & 1.6583 \\
\cline { 2 - 3 } & small & 1.7562 \\
\cline { 2 - 3 } & large & 2.2011 \\
\hline
\end{tabular}

\section{Conclusion}

The problem of optimal path planning for a flexible manipulator has been studied by using the direct process and HS meta-heuristic optimization method in the point to point case and the open-loop mode. The maximum of dynamic load capacity and the minimum transmission time as two criterions determining the efficiency of manipulators have been considered as objective functions in path planning of end the effecter. Full dynamics of the manipulator has been studied 
by taking large deformation of the arms into account. By using smooth trapezoidal and cubic Spline profiles as joint path profiles, the planning of the optimal path has changed to a nonlinear optimization problem through the direct process. To solve the problem of nonlinear optimization, HS efficient method has been used as being appropriate for optimization problems with multiple dimensions and having a high speed of convergence. The proposed method is more effective for path planning than indirect methods (optimal control theory). This is because there is no need to linearize and simplify nonlinear equations of motion which enables the dynamics of the system to be considered completely. The limitation of selecting appropriate weight functions does not exist in the proposed method, and there is no need for suitable initial guess to get faster convergence. To evaluate the effectiveness of the proposed method, optimal path planning for a two-link flexible manipulator has been performed for a trajectory between two points given. Also, comparison has been made between the results obtained for the arms of the rigid, small deformation and large deformation models. The results prove that the proposed method has a good compatibility with all models, and is also applicable to multi-link manipulators.

\section{References}

1. Chettibi T., Lehtihet H.E., Haddad M., 2004, Minimum cost trajectory planning for industrial robots, European Journal of Mechanics A/Solids, 23, 703-715

2. Chettibi T., Lemoine P., 2007, Generation of point to point trajectories for robotic manipulators under electro-mechanical constraints, International Review of Mechanical Engineering, IREME, $131-143$

3. Garg D.P., Kumar M., 2002, Optimization techniques applied to multiple manipulators for path planning and torque minimization, Engineering Applications of Artificial Intelligence, 15, 241-252

4. Haddad M., Chettibi T., Hanchi S., Lehtihet H.S., 2005, A new approach for minimum time motion planning problem of wheeled mobile robots, 16th IFAC World Congress, Prague

5. Haddad M., Hanchi S., Lehtihet H.E., 2007, Sub-optimal motion planner of a mobile manipulator with end-effector's specified path, IFToMM World Congress

6. Heidari H., 2011, Maximum allowable dynamic load of flexible manipulators undergoing large deformation, PhD Thesis, Iran University of Science and Technology, Iran

7. Korayem M.H., Nikoobin A., 2009, Formulation and numerical solution of robot manipulators in point-to-point motion with maximum load carrying capacity, Robotica, 16, 101-109

8. Korayem M.H., Nikoobin A., Azimirad V., 2009, Maximum load carrying capacity of mobile manipulators: Optimal control approach, Robotica, 27, 147-159

9. Korayem M.H., Rahimi Nohooji H., Nikoobin A., 2011, Path planning of mobile elastic robotic arms by indirect approach of optimal control, International Journal of Advanced Robotic Systems, 8, 10-20

10. Piazzi A., Visioli A., 2000, Global minimum-jerk trajectory planning of robot manipulators, IEEE Transactions on Industrial Electronics, 47, 140-149

11. Saravanan R., Ramabalan S., 2008, Evolutionary minimum cost trajectory planning for industrial robots, Journal of Intelligent and Robotic Systems, 52, 45-77

12. Saravanan R., Ramabalan S., Balamurugan C., 2008, Evolutionary optimal trajectory planning for industrial robot with payload constraints, International Journal of Advanced Manufacturing Technology, 38, 1213-1226

13. Spangelo I., Egeland O., 1992, Generation of energy-optimal trajectories for an autonomous underwater vehicle, Proceedings of the 1992 IEEE International Conference on Robotics and Automation, Nice, France, May 12-14 
14. Tangpattanakul P., Artrit P., 2009, Minimum-time trajectory of robot manipulator using Harmony Search algorithm, 6th International Conference on Electrical Engineering/Electronics, Computer, Telecommunication and Information Technology (ECTICON), Chonburi, Thailand

15. Tangpattanakul P., Meesomboon A., Artrit P., 2010, Optimal trajectory of robot manipulator using Harmony search algorithms, SCI, 270, 23-36

16. Zanotto V., Gasparetto A., Lanzutti A., Boscariol P., Vidoni R., 2011, Experimental validation of minimum time-jerk algorithms for industrial robots, Journal of Intelligent and Robotic Systems, 64, 197-219

Manuscript received January 5, 2015; accepted for print July 19, 2015 